Case Report

\title{
Hip Impingement after Anterior Inferior Iliac Spine Avulsion Fractures: A Case Report with Review of the Literature
}

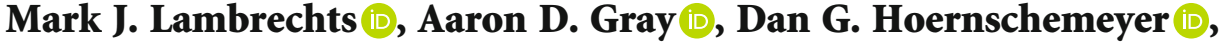 \\ and Sumit Kumar Gupta
}

University of Missouri Department of Orthopaedic Surgery, Columbia, MO, USA

Correspondence should be addressed to Mark J. Lambrechts; lambrechtsm@health.missouri.edu

Received 6 May 2020; Revised 9 September 2020; Accepted 13 October 2020; Published 21 October 2020

Academic Editor: Georg Singer

Copyright (c) 2020 Mark J. Lambrechts et al. This is an open access article distributed under the Creative Commons Attribution License, which permits unrestricted use, distribution, and reproduction in any medium, provided the original work is properly cited.

\begin{abstract}
Avulsion fractures of the anterior inferior iliac spine (AIIS) are rare injuries in adolescent athletes. We present a case of a 15-yearold male who sustained an avulsion injury to his right AIIS when kicking a soccer ball. The patient had chronic pain and extraarticular subspinal impingement leading to decreased hip flexion and rotation. The injury occurred 1.5 years prior to symptom onset, and we were the first health care providers to manage the injury. We attempted six months of nonoperative management including activity modifications and nonsteroidal anti-inflammatory (NSAID) therapy without improvement. Although this injury can often be managed nonoperatively, his symptoms required excision of the AIIS and associated heterotopic ossification. He had an excellent outcome with return to soccer and no pain at his final follow-up visit two years after surgery. Due to the limited literature guiding the surgeon's management of AIIS avulsion injuries with associated heterotopic ossification, we provide a review of the literature detailing pre- and postoperative ranges of motion, surgical approach, fixation or excision of the avulsion fragment, and return to sport in this patient population.
\end{abstract}

\section{Introduction}

Avulsion fractures of the adolescent pelvis are a well-known phenomenon, but the incidence is rare. High-level adolescent soccer players, track and field athletes, gymnasts, and tennis players are known to be at high risk for these injuries [1]. The site of avulsion also appears to be dependent on the particular sport being played. Soccer players are at high risk of avulsion fractures of the anterior inferior iliac spine (AIIS) due to the forceful contracture of the rectus femoris during hip flexion and knee extension when kicking. In fact, approximately $50 \%$ of AIIS avulsion fractures are due to kicking [2]. These fractures can be difficult to diagnose since they are often not visible on standard pelvis X-rays, and a high degree of clinical suspicion is required. Although previous case reports have demonstrated AIIS avulsion fractures months to years after the injury with excessive callus formation and minor decreases in range of motion, other patients have severely limited motion [3, 4]. A combination of the failure to diagnose an acute pelvic avulsion fracture with $\mathrm{X}$-ray and the acquired heterotopic ossification, which can mimic other more dire diagnoses, makes an initial diagnosis of the pelvic avulsion fracture integral in properly treating these patients [5].

The patient and his parents gave informed consent to publish this case report.

\section{Case Report}

A 15-year-old male presented to the clinic with a complaint of right hip pain and decreased range of motion of his hip. A detailed history was performed, which included the patient recalling an injury 18 months prior when he was playing in a nonorganized soccer match. He felt a muscle pull in his right groin. He took a break from playing but resumed after a 10minute rest. Due to continued pain he eventually stopped 


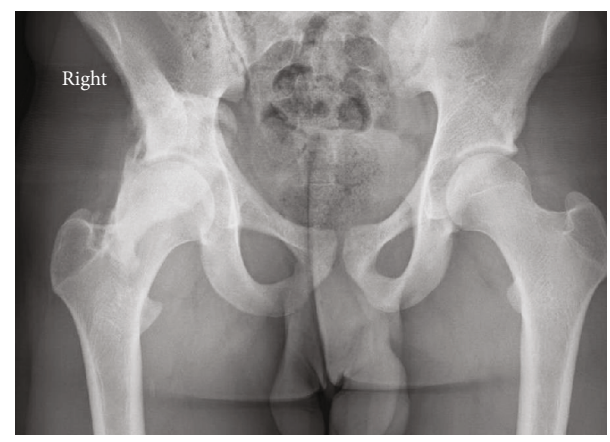

FIGURE 1: AP X-ray of the pelvis demonstrating an avulsion fracture of the right AIIS with large amount of bony callus.

playing for the day. Over the next year, he noticed an increased difficulty with hip flexion especially with squatting and kicking a soccer ball with an associated increase in pain. He presented to our clinic for evaluation 18 months after the initial injury. We were the first doctors to see him regarding his right hip injury.

On physical examination, there was a large hard mass in the area of his right groin. His hip flexion was limited to 70 degrees on the injured extremity compared to 120 degrees on the contralateral side. He had 0 degrees of external rotation. However, he still had 40 degrees of internal rotation.

Imaging with X-rays was remarkable for a large heterotopic bone mass at the AIIS that extended $117 \mathrm{~mm}$ distal to the AIIS and a maximum lateral distance of $64 \mathrm{~mm}$ (Figure 1). Computed tomography (CT) imaging was obtained, which confirmed our suspicion of the diagnosis of an AIIS avulsion fracture with a $117 \mathrm{~mm} \times 65 \mathrm{~mm}$ bony "mass" (Figures 2 and 3). At this time, his diagnosis was consistent with extra-articular subspinal impingement. The patient failed 6 months of conservative management including physical therapy, activity modification, and nonsteroidal anti-inflammatory drugs (NSAIDs). Therefore, surgical treatment for excision of the mass was offered.

We proceeded to the operating theater and removed the heterotopic ossification through an anterior Smith-Peterson approach to the hip (Figure 4). We found that a large part of the origination of the rectus femoris was still attached to the distal aspect of the mass. This was detached and tenodesed. Postoperatively, the patient was placed on a course of indomethacin for 1 month. He returned to playing soccer after his two-month follow-up visit. The postoperative $\mathrm{X}$ rays at one-year follow-up demonstrated no new bone deposition (Figure 5). He had 120-degree hip flexion and full internal and external ranges of motion at his two-year follow-up visit. Importantly, the patient rated his pain at $0 / 10$ at his 2-month, 12-month, and 24-month postoperative visits.

\section{Discussion}

Avulsion fractures of the pelvis are often due to an atraumatic event during sports [6]. Sprinting and kicking are often the inciting events due to forceful contractions of the rectus femoris and hamstring muscles, respectively. Avulsion frac-

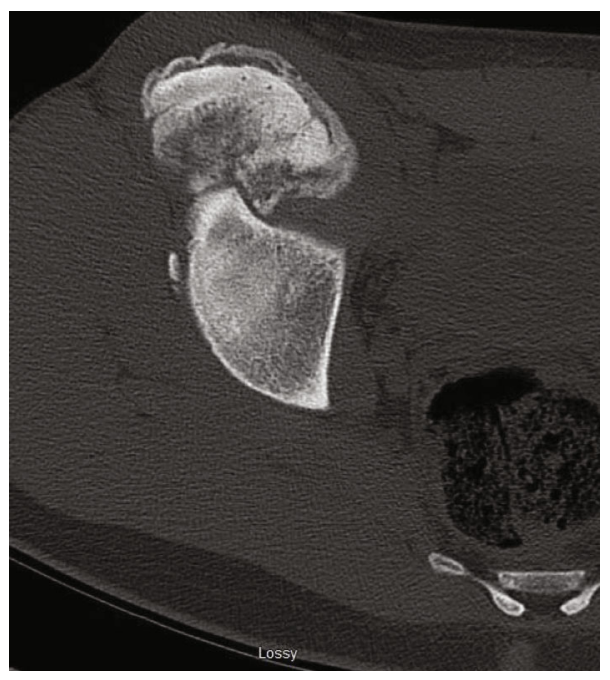

FIgure 2: Axial CT scan demonstrating the large amount of bone deposit from the right AIIS avulsion fracture.

tures often occur in adolescents due to the apophysis being the biomechanical weak link in the tendon-bone kinetic chain [7]. In adults, these forceful contractions lead to muscle strains or tears since the apophysis has closed.

The management of these injuries has been predominantly nonoperative. Return to sports with nonoperative treatment is usually within 2 to 3 months when following the protocol of Metzmaker and Pappas [8]. This includes rest, ice, and NSAIDs for the first week, gentle passive range of motion and assisted weight bearing during week two, resistance training in the third and fourth weeks, and sports-specific training and exercises 4 to 8 weeks after injury. After 2 months, return to sport is usually allowed. However, AIIS fractures are at increased risk of pain with those greater than $2 \mathrm{~cm}$ displaced also at greater risk of nonunion [9]. Large amounts of heterotopic ossification causing symptomatic hip impingement and unremitting pain indicate failures of nonoperative treatment and require operative intervention [10].

Currently, there is some debate whether operative or nonoperative treatment of acute AIIS avulsion fractures has equivalent outcomes. Generally, close follow-up with nonoperative management is an appropriate treatment choice. However, a recent meta-analysis of 596 patients has shown superior outcomes with operative management. Return to sport was $92 \%$ in those treated operatively versus $80 \%$ in nonoperatively treated patients, and those with fractures displaced greater than $15 \mathrm{~mm}$ and elite-level athletes should be considered relative indications to forgo a trial of nonoperative treatment [11].

We performed a literature review to identify all patients requiring surgical intervention of an AIIS avulsion fracture treated nonoperatively with associated heterotopic ossification. Only articles in the English language were considered for inclusion. The following MeSH words were linked "anterior inferior iliac spine" OR “AIIS” AND “avulsion." We identified 8 manuscripts accounting for 9 cases of failed conservative management due to femoroacetabular 


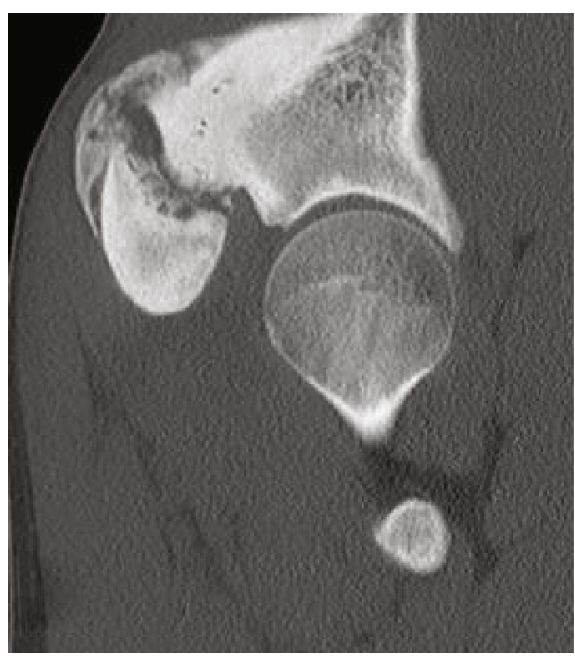

(a)

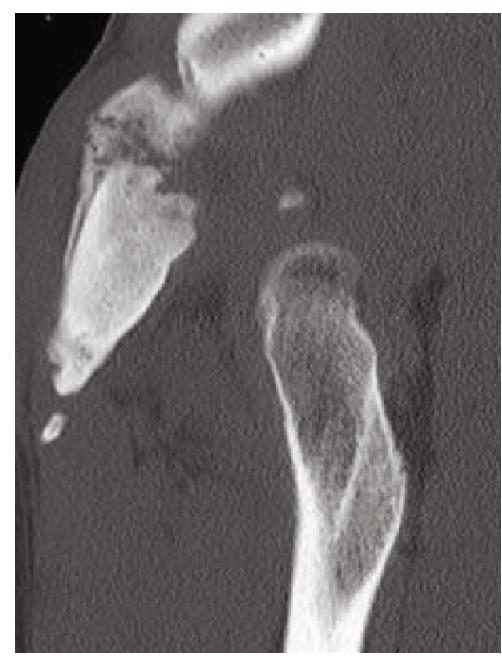

(b)

FIgURE 3: (a) Coronal CT image demonstrating the right AIIS and the large amount of heterotopic ossification. (b) Another sequence from the coronal CT imaging demonstrating the extent of bone growth.

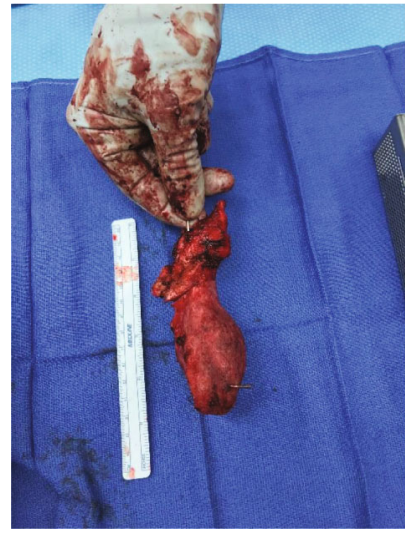

Figure 4: Picture demonstrating actual size $(117 \mathrm{~mm} \times 65 \mathrm{~mm})$ of the heterotopic ossification.

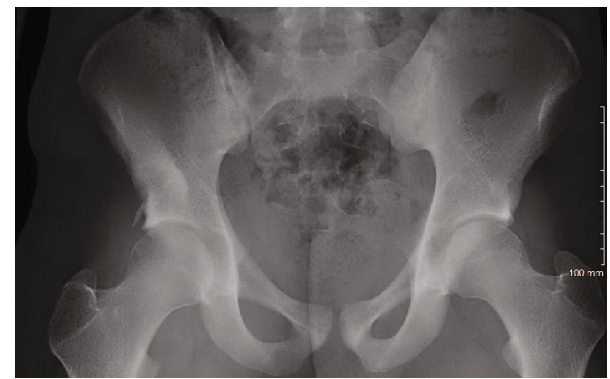

Figure 5: Postoperative X-ray 1 year after heterotopic bone removal.

impingement, pain with hip flexion, or subspinal impingement and reviewed the type of surgical approach, time to return to sport, and improvement in range of motion [4, $10,12-17]$. We believe the following questions are relevant for guiding future research: (1) Is arthroscopic or open exci- sion of heterotopic ossification superior, (2) Is fixation of the fragment superior to excision, (3) Is there an improvement in range of hip motion after surgery, and (4) Do symptoms leading to surgical treatment affect patient outcomes (Table 1)? Due to the limited number of cases reported in the literature, none of these questions can be answered using evidence-based practice at this time. Additionally, large case series including preoperative and postoperative range of motion, reason for operative intervention, type of surgical approach, and time to return to sport should be documented for each reported case to improve treatment in this cohort of patients.

We present this case to highlight the importance of identifying atraumatic injuries of the pelvis in adolescent athletes. If there is suspicion of a pelvic avulsion fracture after physical exam, X-rays should be obtained and a follow-up visit should be ordered. Although an initial clinic visit should be within a month to determine if the patient is improving, it would be reasonable to set a second visit at 4 months as heterotopic ossification is almost universally identified by this time [18]. Parents can be informed that they may call to cancel the appointment if the patient is asymptomatic and back to sporting events. If the pelvic injury is chronic and the patient history and X-ray appear to be inconsistent with a history of an avulsion fracture, a CT scan is the preferred next test. This will identify heterotopic ossification and exclude unlikely differential diagnoses [19].

\section{Conclusion}

Adolescent anterior iliac spine fractures are uncommon injuries during sporting events caused by the apophysis being the weak point of the muscle-tendon junction. AIIS avulsion fractures can be managed nonoperatively if the fracture has minimal displacement and the patient plays sports recreationally. These injuries can cause significant functional 


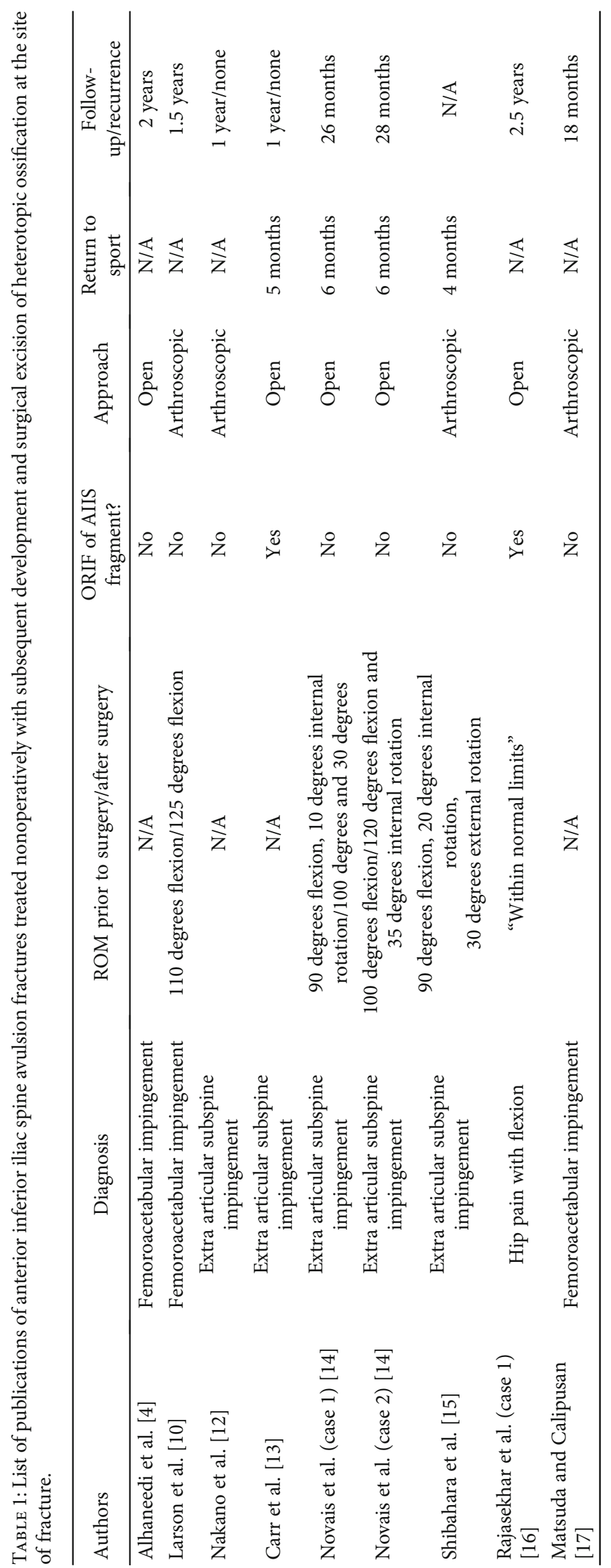


limitations and pain. This necessitates operative management usually resulting in good outcomes.

\section{Conflicts of Interest}

All authors (Dr. M. Lambrechts, Dr. A. Gray, Dr. D. Hoernschemeyer, and Dr. S. Gupta) of this manuscript have no conflict of interest in this article. Neither Dr. Lambrechts nor Dr. Gray report any outside interests. Dr. Hoernschemeyer reports the following disclosures: BioMarin: paid presenter or speaker and research support; OrthoPediatrics: IP royalties; paid consultant; stock or stock options. Dr. Gupta reports the following disclosures: American Academy of Orthopaedic Surgeons: board or committee member; Pediatric Orthopaedic Society of North America: board or committee member.

\section{Authors' Contributions}

MJL, AG, DH, and SKG all took part in preparation of the manuscript. SKG was the attending orthopaedic surgeon and described the surgical technique.

\section{Acknowledgments}

The Barry J. Gainor Resident Research and Scholarship Endowment Fund and the University of Missouri Orthopaedic Association Research Fund provided funding for this study.

\section{References}

[1] F. Rossi and S. Dragoni, "Acute avulsion fractures of the pelvis in adolescent competitive athletes: prevalence, location and sports distribution of 203 cases collected," Skeletal Radiology, vol. 30, no. 3, pp. 127-131, 2001.

[2] D. J. Schuett, J. D. Bomar, and A. T. Pennock, "Pelvic apophyseal avulsion fractures," Journal of Pediatric Orthopedics, vol. 35, no. 6, pp. 617-623, 2015.

[3] D. Pingal, T. Marqueen, and K. Prakash, "Malunited anterior inferior iliac spine fracture as a cause of hip impingement: a case report and review of literature," Chinese Journal of Traumatology, vol. 19, no. 2, pp. 119-121, 2016.

[4] G. A. Alhaneedi, A. S. A. Abdullah, S. I. Ghouri, Y. Abuodeh, and M. M. A. A. Al Dosari, "Avulsion fracture of anterior inferior iliac spine complicated by hypertrophic malunion causing femoroacetabular impingement: case report," International Journal of Surgery Case Reports, vol. 11, pp. 117-120, 2015.

[5] B. S. Dhinsa, A. Jalgaonkar, B. Mann, S. Butt, and R. Pollock, "Avulsion fracture of the anterior superior iliac spine: misdiagnosis of a bone tumour," Journal of Orthopaedics and Traumatology, vol. 12, no. 3, pp. 173-176, 2011.

[6] J. Porr, C. Lucaciu, and S. Birkett, "Avulsion fractures of the pelvis - a qualitative systematic review of the literature," Journal of the Canadian Chiropractic Association, vol. 55, no. 4, pp. 247-255, 2011.

[7] E. J. Vandervliet, F. M. Vanhoenacker, A. Snoeckx, J. L. Gielen, P. van Dyck, and P. M. Parizel, "Sports-related acute and chronic avulsion injuries in children and adolescents with special emphasis on tennis," British Journal of Sports Medicine, vol. 41, no. 11, pp. 827-831, 2007.
[8] J. N. Metzmaker and A. M. Pappas, "Avulsion fractures of the pelvis," The American Journal of Sports Medicine, vol. 13, no. 5, pp. 349-358, 1985.

[9] B. I. McKinney, C. Nelson, and W. Carrion, "Apophyseal avulsion fractures of the hip and pelvis," Orthopedics, vol. 32, no. 1, pp. 42-48, 2009.

[10] C. M. Larson, B. Y. Kelly, and R. M. Stone, "Making a case for anterior inferior iliac spine/subspine hip impingement: three representative case reports and proposed concept," Arthroscopy, vol. 27, no. 12, pp. 1732-1737, 2011.

[11] H. Eberbach, L. Hohloch, M. J. Feucht, L. Konstantinidis, N. P. Südkamp, and J. Zwingmann, "Operative versus conservative treatment of apophyseal avulsion fractures of the pelvis in the adolescents: a systematical review with meta-analysis of clinical outcome and return to sports," BMC Musculoskeletal Disorders, vol. 18, no. 1, p. 162, 2017.

[12] N. Nakano, L. Lisenda, and V. Khanduja, "Arthroscopic excision of heterotopic ossification in the rectus femoris muscle causing extra-articular anterior hip impingement," SICOT-J, vol. 4, p. 41, 2018.

[13] J. B. Carr 2nd, E. Conte, E. A. Rajadhyaksha, K. A. Laroche, F. W. Gwathmey, and E. W. Carson, "Operative fixation of an anterior inferior iliac spine apophyseal avulsion fracture nonunion in an adolescent soccer player," JBJS Case Connector, vol. 7, no. 2, article E29, p. e29, 2017.

[14] E. N. Novais, M. F. Riederer, and A. J. Provance, "Anterior inferior iliac spine deformity as a cause for extra-articular hip impingement in young athletes after an avulsion fracture: a case report," Sports Health: A Multidisciplinary Approach, vol. 10, no. 3, pp. 272-276, 2018.

[15] M. Shibahara, Y. Ohnishi, E. Honda, D. K. Matsuda, and S. Uchida, "Arthroscopic treatment of a displaced nonunion of the anterior inferior iliac spine causing extra-articular impingement," Orthopedics, vol. 40, no. 4, pp. e725-e728, 2017.

[16] C. Rajasekhar, K. Sampath Kumar, and M. S. Bhamra, “Avulsion fractures of the anterior inferior iliac spine: the case for surgical intervention," International Orthopaedics, vol. 24, no. 6, pp. 364-365, 2001.

[17] D. K. Matsuda and C. P. Calipusan, "Adolescent femoroacetabular impingement from malunion of the anteroinferior iliac spine apophysis treated with arthroscopic spinoplasty," Orthopedics, vol. 35, no. 3, pp. e460-e463, 2012.

[18] L. Vanden Bossche and G. Vanderstraeten, "Heterotopic ossification: a review," Journal of Rehabilitation Medicine, vol. 37, no. 3, pp. 129-136, 2005.

[19] M. Incedayi, S. Ozyurek, S. Aribal, K. Keklikci, and G. Sonmez, "Avulsion fracture of the anterior inferior iliac spine mimicking a bone tumor: a case report," Oman Medical Journal, vol. 29 , no. 3, pp. 220-222, 2014. 